\title{
REES MATRIX SEMIGROUPS AND THE REGULAR SEMIDIRECT PRODUCT
}

\author{
K. AUINGER and M. B. SZENDREI ${ }^{\square}$
}

(Received 30 May 2002; revised 18 March 2004)

\author{
Communicated by D. Easdown
}

\begin{abstract}
A generalization of the Pastijn product is introduced so that, on the level of e-varieties and pseudoe-varieties, this product and the regular semidirect product by completely simple semigroups 'almost always' coincide. This is applied to give a model of the bifree objects in every e-variety formed as a regular semidirect product of a variety of inverse semigroups by a variety of completely simple semigroups that is not a group variety.
\end{abstract}

2000 Mathematics subject classification: primary 20M17, 20M07, 20M10.

\section{Introduction}

Semidirect products appeared in the theory of regular semigroups in the 1970's with H. E. Scheiblich's model of free inverse semigroups and D. B. McAlister's results on $E$-unitary inverse semigroups. While a semidirect product of a regular semigroup by a group is necessarily regular, this is far from being the case in general when the group is replaced by a regular semigroup. This 'defect' led to the development of several variants, each generalizing the semidirect product by groups and producing regular semigroups from regular ones.

Let us mention here three of them, each of which is defined for any regular semigroup in the first factor and any completely simple semigroup in the second. The

The authors gratefully acknowledge support from the Austro-Hungarian Bilateral Intergovernmental Cooperation project No. A-24/2000.

The second author was partially supported by the Hungarian National Foundation for Scientific Research, Grant No. T26243 and T34175, and by the Ministry of Education, Grant No. FKFP1030. (C) 2005 Australian Mathematical Society 1446-7887/05 $\$$ A2.00+0.00 
restricted regular semidirect product was introduced by Polák and the first author in [2]. The Pastijn product originates in [17] and was applied by Kadourek [10] to describe the bifree objects in certain e-varieties of locally inverse semigroups, and so to provide decompositions of these e-varieties as a Pastijn product of the variety of semilattices by a variety of completely simple semigroups. Further decomposition results using the Pastijn product can be found in [11] and [12]. The notion of the regular semidirect product is due to Jones and Trotter in [9] where, among a great number of decompositions of certain e-varieties as regular semidirect products, special attention is paid to those where the second factor is the variety of right zero semigroups. For a remarkable result in this direction, see [8].

It was noticed by Billhardt and the second author in [5] that, for any (regular) semigroup $T$ and completely simple semigroup $C$, each restricted regular semidirect product of $T$ by $C$ is isomorphic to a Pastijn product of a (regular) subsemigroup of $T$ by $C$, and, conversely, each Pastijn product of $T$ by $C$ is embeddable into a restricted regular semidirect product of a direct power of $T$ by $C$. Therefore the restricted regular semidirect product by a completely simple semigroup and the Pastijn product coincide on the level of e-varieties and pseudo-e-varieties.

The regular semidirect product by a completely simple semigroup is a more general construction: for any regular semigroup $T$ and completely simple semigroup $C$ acting on $T$, the restricted regular semidirect product of $T$ by $C$ is a subsemigroup of the regular semidirect product of $T$ by $C$. On the other hand, the Pastijn product of $T$ by $C$ is a subsemigroup of a regular Rees matrix semigroup over a semidirect product of $T^{1}$ by a maximal subgroup of $C$ where the entries of the sandwich matrix have a special form. The aim of this paper is to show that the regular semidirect product by a completely simple semigroup and the generalization of the Pastijn product obtained by omitting the restriction on the entries of the sandwich matrix - called Pastijn-Rees product - 'almost always' coincide on the level of e-varieties and pseudo-e-varieties (Section 3). This result allows us to give a model of the bifree objects in any e-variety which is a regular semidirect product of a variety of inverse semigroups by a variety of completely simple semigroups that is not a group variety (Section 4).

The results of this paper have been obtained while investigating how far the restricted regular semidirect product and the regular semidirect product of the variety of semilattices by a variety of completely simple semigroups can be from each other. The results in this direction are published in [3].

\section{Preliminaries}

For the basic notions and notation in semigroup theory, the reader is referred to [7]. Let $S$ and $T$ be semigroups. If $S$ is regular and $T$ is a homomorphic image of a 
regular subsemigroup of $S$ then we say that $T$ regularly divides $S$. We use $\operatorname{Reg}(S)$ to denote the set of all regular elements in $S$ and End $S$ the endomorphism monoid of $S$.

Let $I$ and $\Lambda$ be non-empty sets. Consider a $\Lambda \times I$ matrix $P=\left(p_{\lambda i}\right)$ over $S$. The $I \times \Lambda$ Rees matrix semigroup over $S$ with sandwich matrix $P$, denoted $\mathcal{M}(I, S, \Lambda ; P)$, is the set $I \times S \times \Lambda$ endowed with the following multiplication:

$$
(i, s, \lambda)(j, t, \mu)=\left(i, s p_{\lambda j} t, \mu\right) .
$$

It is well known that if $S$ is a group then $\mathcal{M}(I, S, \Lambda ; P)$ is completely simple and, conversely, each completely simple semigroup is isomorphic to a Rees matrix semigroup over a group. Therefore we refer to Rees matrix semigroups over groups also as completely simple semigroups.

Completely simple semigroups are regular but, in general, a Rees matrix semigroup over a regular semigroup need not be regular. However, the set of regular elements forms a (regular) subsemigroup. This crucial observation is due to McAlister [15].

RESULT 2.1. Let $S$ be a regular semigroup, $I$ and $\Lambda$ be non-empty sets and let $P=\left(p_{\lambda i}\right)$ be a $\Lambda \times I$ matrix over $S$. Then

(i) an element $(i, s, \lambda) \in \mathcal{M}(I, S, \Lambda ; P)$ is idempotent if and only if $s=s p_{\lambda i}$;

(ii) an element $(i, s, \lambda) \in \mathcal{M}(I, S, \Lambda ; P)$ is regular if and only if $V(s) \cap p_{\lambda j} S p_{\mu i} \neq$ $\emptyset$ for some $j \in I$ and $\mu \in \Lambda$;

(iii) the set $\operatorname{Reg}(\mathcal{M}(I, S, \Lambda ; P))$ of regular elements of $\mathcal{M}(I, S, \Lambda ; P)$ forms a (regular) subsemigroup of $\mathcal{M}(I, S, \Lambda ; P)$.

The subsemigroup $\operatorname{Reg}(\mathcal{M}(I, S, \Lambda ; P))$ is usually denoted $\mathcal{R} \mathcal{M}(I, S, \Lambda ; P)$ and is called the $I \times \Lambda$ regular Rees matrix semigroup over $S$ with sandwich matrix $P$.

Regular Rees matrix semigroups over inverse semigroups are of special importance (see $[15,16])$.

RESULT 2.2. Each regular Rees matrix semigroup over an inverse semigroup is locally inverse.

Result 2.1 (ii) does not provide an inverse of a regular element of $\mathcal{M}(I, S, \Lambda ; P)$. Therefore we give an alternative characterization of regularity, and produce inverses, the proofs of which are routine and omitted.

PROPOSITION 2.3. Let $S$ be a regular semigroup, $I$ and $\Lambda$ be non-empty sets and let $P=\left(p_{\lambda i}\right)$ be a $\Lambda \times I$ matrix over $S$. Then an element $(i, s, \lambda) \in \mathcal{M}(I, S, \Lambda ; P)$ is regular if and only if there exists $j \in I$ such that $s p_{\lambda j} \mathcal{R} s$ and $\mu \in \Lambda$ such that $p_{\mu i} s \mathcal{L} s$, in which case $\left(j,\left(s p_{\lambda j}\right)^{\prime} s\left(p_{\mu i} s\right)^{\prime}, \mu\right) \in V((i, s, \lambda))$ for any $\left(s p_{\lambda j}\right)^{\prime} \in V\left(s p_{\lambda j}\right)$ and $\left(p_{\mu i} s\right)^{\prime} \in V\left(p_{\mu i} s\right)$. 
By a (left) action of $T$ on $S$ we mean an antihomomorphism $\varepsilon: T \rightarrow$ End $S, t \mapsto \varepsilon_{t}$ such that $\varepsilon_{1}$ is the identity automorphism provided $T$ is a monoid. For brevity, we denote $a \varepsilon_{t}$ by ' $a(a \in S, t \in T)$. If $T$ is a monoid then ${ }^{1} a=a$ for every $a \in S$. In particular, if $T$ is a group then $\varepsilon_{t}$ is an automorphism for every $t \in T$. Note that, in [9], the condition on $\varepsilon_{1}$ is not required, and an action with this additional property is termed left unitary.

The semidirect product $S * T$ of $S$ by $T$ with respect to this action is defined on the set $S \times T$ by the multiplication

$$
(a, t)(b, u)=(a \cdot b, t u) \quad(a, b \in S, t, u \in T) .
$$

A straightforward calculation shows that $S * T$ is a semigroup.

If $A, B$ are non-empty sets then the set of all mappings of $A$ into $B$ is denoted by $B^{A}$. Also $S^{A}$ stands for the direct power of $S$ to the exponent $A$, and the product of the elements $f, g$ in the semigroup $S^{A}$ is denoted by $f g$.

The wreath product of $S$ by $T$, denoted by $S$ wr $T$, is the semidirect product of $S^{T^{1}}$ by $T$ with respect to the following action: for every $f \in S^{T^{1}}$ and $t \in T$, we define 'f $\in S^{T^{1}}$ by $x\left({ }^{\prime} f\right)=(x t) f\left(x \in T^{1}\right)$. Notice that if $T$ has an identity then indeed ${ }^{1} f=f$ for every $f \in S^{T^{1}}$. Note that there is a natural embedding of any semidirect product $S * T$ into $S$ wr $T$ :

$$
\nu: S * T \rightarrow S \text { wr } T, \quad(a, t) \nu=\left(f_{a}, t\right) \quad(a \in S, t \in T),
$$

where

$$
f_{a}: T^{1} \rightarrow S, \quad x f_{a}={ }^{x} a \quad\left(x \in T^{1}\right) .
$$

A semidirect or wreath product of regular semigroups need not be regular. However, a regular version of the semidirect product was introduced in [9] as follows. It was noticed that if $S$ and $T$ are regular, $T$ acts on $S$ and at least one of $S$ and $T$ is completely simple then $\operatorname{Reg}(S * T)$ forms a (regular) subsemigroup in $S * T$, and the regular semidirect product $S * r, T$ of $S$ by $T$ was defined to be $\operatorname{Reg}(S * T)$. In particular, $\operatorname{Reg}(S w r T)$ is a regular subsemigroup in $S$ wr $T$, which we denote by $S$ wrr $T$. Since $v$ preserves regularity, $S *_{r} T$ embeds into $S$ wrr $T$. Note that if $S$ or $T$ is a group then $S *, T=S * T$ and $S$ wrr $T=S$ wr $T$. In this paper, we are interested in regular semidirect products of regular semigroups by completely simple semigroups.

The following result from [4] describes the regular elements of semidirect products of regular semigroups, and gives inverses.

RESULT 2.4. Let $S$ and $T$ be regular semigroups, and let $T$ act on $S$. An element $(a, t) \in S * T$ is regular if and only if there exists $t^{\prime} \in V(t)$ such that " $a \geq_{\mathcal{L}} a$ in $S$. If this is the case then $\left({ }^{\prime} a^{\prime}, t^{\prime}\right) \in V((a, t))$ for any $a^{\prime} \in V(a)$. 
A special role will be played in the paper by semidirect products of inverse semigroups by groups which are known to be inverse semigroups, and their basic properties can be found in [14].

Following Hall [7], a class of regular semigroups is termed an existence variety, or briefly an $e$-variety if it is closed under the operators $\mathbf{P}, \mathbf{S}_{r}$ and $\mathbf{H}$ of forming direct products, regular subsemigroups and homomorphic images, respectively. In particular, a class of inverse semigroups or of completely simple semigroups constitutes an evariety if and only if it is a variety of unary semigroups in the usual sense. Therefore, for classes of inverse semigroups and of completely simple semigroups, we will use the term 'variety' rather than 'e-variety'. We introduce notation for the following e-varieties:

$$
\begin{aligned}
& \mathcal{L Z} \text { - left zero semigroups, } \\
& \mathcal{R B} \text { - rectangular bands, } \\
& \mathcal{G} \text { - groups, } \\
& \mathcal{C S} \text { - completely simple semigroups, }
\end{aligned}
$$

$$
\begin{aligned}
& \mathcal{R Z} \text { - right zero semigroups, } \\
& \mathcal{S} \text { - semilattices, } \\
& \mathcal{I} \text { - inverse semigroups, } \\
& L \mathcal{I} \text { - locally inverse semigroups. }
\end{aligned}
$$

The finitary analogue of an e-variety is a pseudo-e-variety, which is defined to be a class of finite regular semigroups closed under forming finite direct products, regular subsemigroups and homomorphic images.

Let $X$ be a non-empty set. We 'double' it in the following way: consider a set $X^{\prime}$ disjoint from $X$ together with a bijection ': $X \rightarrow X^{\prime}, x \mapsto x^{\prime}$, and put $\bar{X}=X \cup X^{\prime}$. Given a regular semigroup $S$, a mapping $\vartheta: \bar{X} \rightarrow S$ is called matched if $x \vartheta, x^{\prime} \vartheta$ are mutual inverses in $S$ for all $x \in X$. Let $\mathcal{C}$ be a class of regular semigroups. A bifree object in $\mathcal{C}$ on a non-empty set $X$ is defined to be a semigroup $S \in \mathcal{C}$ together with a matched mapping $\iota: \bar{X} \rightarrow S$ satisfying the following universal property: for any semigroup $T \in \mathcal{C}$ and any matched mapping $\vartheta: \bar{X} \rightarrow T$, there exists a unique homomorphism $\varphi: S \rightarrow T$ such that $\iota \varphi=\vartheta$. Obviously, such a bifree object is unique up to isomorphism, if it exists.

It was proved by Yeh [19] that each e-variety of locally inverse semigroups has a bifree object on any non-empty set. In particular, in a variety of inverse semigroups, the bifree objects coincide with the free objects. However, in a variety of completely simple semigroups, the free object on a set is, up to isomorphism, a proper subsemigroup in the bifree object on the same set. The reason for the existence of the bifree objects in e-varieties of locally inverse semigroups is the following crucial property of locally inverse semigroups. For every locally inverse semigroup $S$ and for every subset $U$ of $S$ such that each element of $U$ has an inverse belonging to $U$, there exists a least regular subsemigroup in $S$ containing $U$. This subsemigroup is the least subsemigroup in $S$ containing $U$ and being closed under forming sandwich elements (the $\wedge$ operation), and it is called the regular subsemigroup in $S$ generated 
by $U$. An important consequence of this fact is that, for any class $\mathcal{C}$ of locally inverse semigroups, the e-variety generated by $\mathcal{C}$ is $\mathbf{H S}, \mathbf{P C}$ (see [19]).

Given a variety $\mathcal{V}$ of inverse semigroups and a non-empty set $X$, we denote by $F \mathcal{V}(X)$ the model $\mathbf{I}(X) / \rho_{\mathcal{V}}$ of the free object in $\mathcal{V}$ on $X$ where $\mathbf{I}(X)$ is the free semigroup with involution on $X$ and $\rho_{\mathcal{V}}$ is the fully invariant congruence on $\mathbf{I}(X)$ corresponding to $\mathcal{V}$ (see [18]). The underlying semigroup of $I(X)$ is the free semigroup on $\bar{X}$, and the unary operation ${ }^{-1}$ is the extension of the bijection ' $: X \rightarrow X^{\prime}$ to an involutory antiautomorphism. For notational convenience, we write ' -1 ' instead of " ", and suppose that $\bar{X} \subseteq F \mathcal{V}(X)$.

A similar description of bifree objects in e-varieties of locally inverse semigroups was given in [1]. In this generality, the semigroup of terms $\mathbf{T}(X)$ is the free binary semigroup on $\bar{X}$, that is, the absolutely free algebra on $\bar{X}$ in two binary operations . and $\wedge$, where the operation $\cdot$ is assumed to be associative. Although we do not need it explicitly in this paper, we mention for completeness that, for any e-variety $\mathcal{V}$ of locally inverse semigroups and any non-empty set $X$, the bifree object in $\mathcal{V}$ on $X$ can be obtained as a factor semigroup of $\mathbf{T}(X)$ modulo a congruence, called the bi-invariant congruence, on $\mathbf{T}(X)$ corresponding to $\mathcal{V}$.

Now let $\mathcal{V}$ be a variety of completely simple semigroups with $\mathcal{R B} \subseteq \mathcal{V}$, and let $X$ be a non-empty set. We denote by $B F \mathcal{V}(X)$ the Rees matrix model of the bifree object in $\mathcal{V}$ on $X$ obtained as follows (see [10]). Consider $C=\mathcal{M}\left(\bar{X}, G_{\mathcal{V}}, \bar{X} ; P\right)$, where $P$ is an $\bar{X} \times \bar{X}$ matrix normalized at $x_{0} \in X$, and such that $p_{y z} \neq p_{u v}$ if $(y, z) \neq(u, v)$ in $\left(\bar{X} \backslash\left\{x_{0}\right\}\right) \times\left(\bar{X} \backslash\left\{x_{0}\right\}\right), \hat{P}=\left\{p_{y z}: y, z \in \bar{X} \backslash\left\{x_{0}\right\}\right\}, G$ is the free group on $X \cup \hat{P}$ and $G_{\mathcal{V}}=G / N_{\mathcal{V}}$ where the normal subgroup $N_{\mathcal{V}}$ is determined by $\mathcal{V}$. For simplicity, we suppose that $X \cup \hat{P} \subseteq G_{\mathcal{V}}$. The matched mapping corresponding to $C$ is $\bar{X} \rightarrow C$, $x \mapsto c_{x}=(x, x, x), x^{\prime} \mapsto c_{x^{\prime}}=\left(x^{\prime}, p_{x x^{\prime}}^{-1} x^{-1} p_{x^{\prime} x}^{-1}, x^{\prime}\right)$.

As usual, for any $w \in \mathbf{T}(X)$, we denote the element of $C$ corresponding to $w$ by $(h(w), m(w), t(w))$. In particular, we have

$$
\begin{aligned}
& h(x)=x, \quad m(x)=x, \quad t(x)=x \quad \text { and } \\
& h\left(x^{\prime}\right)=x^{\prime}, \quad m\left(x^{\prime}\right)=p_{x x^{\prime}}^{-1} x^{-1} p_{x^{\prime} x}^{-1}, \quad t\left(x^{\prime}\right)=x^{\prime} .
\end{aligned}
$$

Given any e-variety [pseudo-e-variety] $\mathcal{U}$ and a variety [pseudo-e-variety] $\mathcal{V}$ of completely simple semigroups, the regular semidirect product $\mathcal{U} *_{r} \mathcal{V}$ of $\mathcal{U}$ by $\mathcal{V}$ is defined in [9] to be the e-variety [pseudo-e-variety] generated by the class of all regular semidirect products $T *_{r} C$ with $T \in \mathcal{U}$ and $C \in \mathcal{V}$.

\section{The Pastijn-Rees product and its connection to the regular semidirect product}

In this section we define the notion of a (regular) Pastijn-Rees product of a (regular) semigroup by a completely simple semigroup, and investigate the relation between 
the regular semidirect products and the regular Pastijn-Rees products of regular semigroups by completely simple semigroups. As a consequence, we establish that, in most cases, these products coincide on the level of e-varieties and pseudo-e-varieties.

Let $T$ be a semigroup and $G$ a group acting on $T$. Consider a Rees matrix semigroup $C=\mathcal{M}(I, G, \Lambda ; P)$. Let $Q=\left(q_{\lambda i}\right)$ be a $\Lambda \times I$ matrix over the semigroup $T^{1}$. Define a multiplication on the set $T \diamond C=I \times T \times G \times \Lambda$ as follows:

$$
(i, a, g, \lambda)(j, b, h, \mu)=\left(i, a \cdot{ }^{8} q_{\lambda j} \cdot{ }^{8 p^{\lambda j}} b, g p_{\lambda j} h, \mu\right) .
$$

This multiplication is associative, and so $T \diamond C$ a semigroup which is easily seen to be a subsemigroup of a Rees matrix semigroup over a semidirect product as follows. The action of $G$ can be naturally extended to an action of $T^{1}$ by putting ${ }^{8} 1=1$ for every $g \in G$. This defines a semidirect product $T^{1} * G$. We can form a $\Lambda \times I$ matrix $Q \times P$ over $T^{1} * G$ by putting $(Q \times P)_{\lambda i}=\left(q_{\lambda i}, p_{\lambda i}\right)$ for every $\lambda \in \Lambda$ and $i \in I$. Then $T \diamond C$ may be identified with a subsemigroup of $\mathcal{M}\left(I, T^{1} * G, \Lambda ; Q \times P\right)$ since

$$
(a, g)\left(q_{\lambda j}, p_{\lambda j}\right)(b, h)=\left(a \cdot{ }^{8} q_{\lambda j} \cdot{ }^{g p_{\lambda j}} b, g p_{\lambda j} h\right)
$$

in $T^{1} * G$. Moreover, $T \diamond C$ is a co-extension of $C$ via the projection $(i, a, g, \lambda) \mapsto$ $(i, g, \lambda)$. Therefore we term $T \diamond C$ the Pastijn-Rees product of $T$ by $C$ with respect to the action of $G$ on $T$ and the matrix $Q$. If we need to indicate the action $\gamma$ of $G$ on $T$ and the matrix $Q$ then we write $T \diamond^{r . Q} C$. Sometimes, we shall find it convenient to denote an element of the Rees matrix semigroup $C$ with a single letter instead of a triple. Therefore, an element $(i, a, g, \lambda) \in T \diamond C$ with $c=(i, g, \lambda) \in C$ will be also denoted by $(a ; c)$.

ReMARK 3.1. The Pastijn-Rees product is a common generalization of the Rees matrix construction $(T \diamond C=\mathcal{M}(I, T, \Lambda ; Q)$ if $C$ is the rectangular band $I \times \Lambda$ and each member of $Q$ belongs to $T$ ) and the Pastijn product ( $T \diamond C=T \odot C$ if each entry in $Q$ is 1 ). In particular, it is a generalization of a semidirect product by a group ( $T \diamond C=T * C$ if $C$ is a group and the unique entry in $Q$ is 1 ).

It was noticed in [10] that a Pastijn product $T \odot C$ is independent of the choice of the Rees matrix representation of the completely simple semigroup $C$. A similar assertion holds more generally for Pastijn-Rees products.

Proposition 3.2. Let $C=\mathcal{M}(I, G, \Lambda ; P)$ and $D=\mathcal{M}(J, H, \Theta ; R)$ be isomorphic completely simple semigroups and let $T$ be an arbitrary semigroup. Moreover, let $Q$ be a $\Lambda \times I$ matrix over $T^{1}$, and suppose that an action $\gamma$ of $G$ on $T$ is given. Then there exist a $\Theta \times J$ matrix $S$ over $T^{1}$ and an action $\delta$ of $H$ on $T$ such that $T \diamond^{r, Q} C$ is isomorphic to $T \diamond^{\delta, S} D$. 
PROOF. Let $P=\left(p_{\lambda i}\right), Q=\left(q_{\lambda i}\right)$ and $R=\left(r_{\vartheta j}\right)$. It is well known that the isomorphic Rees matrix semigroups $C$ and $D$ relate to each other in the following way: there exist bijections $\phi: I \rightarrow J, \psi: \Lambda \rightarrow \Theta$, an isomorphism $\omega: G \rightarrow H$ and families of elements $u_{i} \in H, i \in I$, and $v_{\lambda} \in H, \lambda \in \Lambda$, such that $p_{\lambda i} \omega=v_{\lambda} r_{\lambda \psi, i \phi} u_{i}$ for every $i \in I$ and $\lambda \in \Lambda$, and the rule $(i, g, \lambda) \mapsto\left(i \phi, u_{i} \cdot g \omega \cdot v_{\lambda}, \lambda \psi\right)$ determines an isomorphism of $C$ onto $D$. Define a $\Theta \times J$ matrix $S=\left(s_{\vartheta j}\right)$ over $T^{1}$ as follows: for every $\vartheta \in \Theta$ and $j \in J$, put

$$
s_{\vartheta j}={ }^{v_{\lambda}^{-1} \omega^{-1}} q_{\lambda i} \text { where } \lambda=\vartheta \psi^{-1} \text { and } i=j \phi^{-1} .
$$

Moreover, define an action $\delta$. of $H$ on $T$ by means of the action $\gamma$ of $G$ on $T$ in the following manner: for any $h \in H$ and $a \in T$, let

$$
{ }^{h} a={ }^{h \omega^{-1}} a .
$$

It is straightforward to check that the mapping $T \diamond^{\gamma, Q} C \rightarrow T \diamond^{\delta, S} D$ defined by

$$
(i, a, g, \lambda) \mapsto\left(i \phi,{ }^{u_{i} \omega^{-1}} a, u_{i} \cdot g \omega \cdot v_{\lambda}, \lambda \psi\right)
$$

is an isomorphism. This completes the proof.

This assertion allows us to speak, in general, about a Pastijn-Rees product by a completely simple semigroup.

Now we establish that each Pastijn-Rees product is embeddable into a semidirect product.

PROPOSITION 3.3. Let $T$ be a semigroup and $C$ a completely simple semigroup which is not a group. Each Pastijn-Rees product of $T$ by $C$ is embeddable into the wreath product of $T$ by $C$, that is, into a semidirect product of a direct power of $T$ by $C$.

Proof. Let $T \diamond C$ be a Pastijn-Rees product of $T$ by $C=\mathcal{M}(I, G, \Lambda ; P)$ with respect to an action of $G$ on $T$ and a matrix $Q=\left(q_{\lambda i}\right)$. For any $i \in l$ and $a \in T$, let us define a mapping $f_{i, a}: C^{1} \rightarrow T$ by $1 f_{i, a}=a$ and $(k, x, \kappa) f_{i, a}={ }^{x} q_{k i} \cdot{ }^{x p_{k i} a}$ for every $(k, x, \kappa) \in C$. This mapping is well defined since $C$ has no identity. It is easy to check that $\iota: T \diamond C \rightarrow T$ wr $C\left(=T^{C^{1}} * C\right),(i, a, g, \lambda) \iota=\left(f_{i, a} ; i, g, \lambda\right)$ is an embedding. This completes the proof.

Parts (i) and (ii) of the following proposition are routine. Part (iii), which says again that regular elements form a subsemigroup, follows easily from Result 2.1 (iii).

PROPOSITION 3.4. Let $T \diamond C=T \diamond^{\gamma, Q} C$ be a Pastijn-Rees product of a regular semigroup $T$ by $C=\mathcal{M}(I, G, \Lambda ; P)$. Then 
(i) an element $(i, a, g, \lambda) \in T \diamond C$ is idempotent if and only if $g=p_{\lambda i}^{-1}$ and $a=a \cdot{ }^{p_{\lambda i}^{-1}} q_{\lambda i} a$;

(ii) an element $(i, a, g, \lambda) \in T \diamond C$ is regular if and only if there exists $j \in I$ such that $a \cdot{ }^{8} q_{\lambda j} \mathcal{R} a$ and there exists $\mu \in \Lambda$ such that ${ }^{P_{\mu i}^{-1}} q_{\mu i} a \mathcal{L} a ;$ if this is the case, then $\left(j,{ }^{p_{\lambda j}^{-1} g^{-1}}\left(\left(a \cdot{ }^{g} q_{\lambda j}\right)^{\prime} a\left({ }^{p_{\mu i}^{-1}} q_{\mu i} a\right)^{\prime}\right), p_{\lambda j}^{-1} g^{-1} p_{\mu i}^{-1}, \mu\right) \in V((i, a, g, \lambda))$ for any $\left(a \cdot{ }^{g} q_{\lambda j}\right)^{\prime} \in$ $V\left(a \cdot{ }^{8} q_{\lambda j}\right)$ and $\left({ }^{p_{\mu i}^{-1}} q_{\mu i} a\right)^{\prime} \in V\left({ }^{p_{\mu i}^{-1}} q_{\mu i} a\right)$;

(iii) the set $\operatorname{Reg}(T \diamond C)$ of regular elements of $T \diamond C$ forms a (regular) subsemigroup in $T \diamond C$.

The regular subsemigroup $\operatorname{Reg}(T \diamond C)$ in $T \diamond C$ will be called a regular Pastijn-Rees product of $T$ by $C$ and denoted by $T \diamond_{r} C$ or $T \diamond_{r}^{\gamma, Q} C$.

For later use, we record the following important observation, which follows easily from Result 2.2.

PROPOSITION 3.5. A regular Pastijn-Rees product of an inverse semigroup by a completely simple semigroup is a locally inverse semigroup.

The following consequence of Propositions 3.3 and 3.4 (iii) can be easily deduced.

COROLlARY 3.6. Let $T$ be a regular semigroup and $C$ a completely simple semigroup which is not a group. Each regular Pastijn-Rees product of $T$ by $C$ is embeddable into the regular wreath product of $T$ by $C$, that is, into a regular semidirect product of a direct power of $T$ by $C$.

Now we find an analogous result for Pastijn-Rees products by groups.

PROPOSITION 3.7. Let $T$ be a regular semigroup and $G$ a group. Each regular Pastijn-Rees product of $T$ by $G$ is embeddable into the direct square of a (regular) semidirect product of $T$ by $G$.

PROOF. If $S$ is a regular semigroup and $u \in S^{1}$ then one can define a multiplication $\circ_{u}$ on the set $S$ by $a \circ_{u} b=a u b$. It is routine to see that $S_{u}=\left(S ; \circ_{u}\right)$ is a semigroup called a variant of $S$ (see [13]). It follows by Proposition 2.3 that $\operatorname{Reg}\left(S_{u}\right)=\{a \in S: a u \mathcal{R} a \mathcal{L} u a\}$, and $\operatorname{Reg}\left(S_{u}\right)$ is a (regular) subsemigroup of $S_{u}$. Let $T \diamond_{r}^{\gamma, Q} G$ be a regular Pastijn-Rees product of $T$ by $G$ where $G$ is regarded trivially as a Rees matrix semigroup over itself (which suffices by Proposition 3.2). Let $S$ be the semidirect product of $T$ by $G$ defined by the action $\gamma$. It is easy to see by the definition of the Pastijn-Rees product that $T \diamond^{\gamma, Q} G=S_{u}$ with $u=(q, 1)$ where $q$ is the unique element of $Q$. Therefore, in order to prove the statement, it suffices to verify that $\operatorname{Reg}\left(S_{u}\right)$ is embeddable into the direct square of $S$. Let us define a mapping $\phi: \operatorname{Reg}\left(S_{u}\right) \rightarrow S \times S$ by $a \phi=(a u, u a)$ which is clearly a homomorphism. If $a, b \in \operatorname{Reg}\left(S_{u}\right)$ and $a \phi=b \phi$ then $a u \mathcal{R} a \mathcal{L} u a, b u \mathcal{R} b \mathcal{L} u b$ and $a u=b u, u a=u b$, 
which immediately implies that $a=b$. Therefore $\phi$ is injective, and the proof is complete.

The main purpose of this section is to find a relation in the opposite direction, that is, to obtain each regular semidirect product from an appropriate regular Pastijn-Rees product.

For semidirect products by groups, this relation is obvious (see Remark 3.1). Now we consider the general case.

PROPOSITION 3.8. Let $T$ be a regular semigroup and $C$ a completely simple semigroup. Each regular semidirect product of $T$ by $C$ regularly divides a regular PastijnRees product $T_{0} \diamond_{r} D$ where $T_{0}$ is a regular subsemigroup of $T$ and $D$ is a subdirect product of $C$ and a left zero semigroup.

ProOF. Assume that $C$ acts on $T$, and let $T *_{r} C$ be defined by this action. We construct a regular subsemigroup $W$ in a direct product of a left zero semigroup $L$ and a regular Pastijn-Rees product $T_{0} \diamond_{r}^{\gamma, Q} D$ where $D$ is a subdirect product of $C$ and a left zero semigroup, such that $T *_{r} C$ is a homomorphic image of $W$. The statement of the proposition follows if we observe that $L \times\left(T_{0} \diamond_{r}^{\gamma, Q} D\right)$ is isomorphic to $T_{0} \diamond_{r}^{r, \tilde{Q}}(L \times D)$, where, if $D=\mathcal{M}(J, G, \Lambda ; P)$, we choose the Rees matrix representation $\mathcal{M}(L \times J, G, \Lambda ; \tilde{P})$ for the direct product $L \times D$ with $\tilde{p}_{\lambda(l, j)}=p_{\lambda j}$ and $\tilde{q}_{\lambda(l, j)}=q_{\lambda j}$ for any $\lambda \in \Lambda$ and $(l, j) \in L \times J$.

Suppose that $C=\mathcal{M}(I, G, \Lambda ; P)$ where $P=\left(p_{\lambda i}\right)$ is normalized at $0 \in I \cap \Lambda$. The rough idea of the construction is the following. The action of $G$ will be the restriction of the action of $C$ to $G$ where $G$ is identified with $G_{0}=\{(0, g, 0) \in C: g \in G\}$, and $T_{0}$ will be the greatest subsemigroup in $T$ on which $G$ acts by automorphisms. The completely simple semigroup $D$ is obtained from $C$ by 'substituting' $I$ by the set of all pairs of mutually inverse elements of $T *_{r} C$. The aim of this 'blowing up' is to ensure that all pairs of mutually inverse elements of the subsemigroup

$$
{ }^{c} T *_{r} C=\left\{\left({ }^{b} t, c\right) \in T *_{r} C: b, c \in C, t \in T\right\}
$$

of $T *_{r} C$ possess inverse images in $T_{0} \diamond^{\gamma, Q} D$ of the form described in Proposition 3.4. Finally, we need to form the direct product $L \times\left(T_{0} \diamond_{r}^{\gamma, Q} D\right)$, where $L$ will actually be the left zero semigroup on the set $T$, in order that all pairs of mutually inverse elements of $T *, C$ have inverse images in $L \times\left(T_{0} \diamond_{r}^{\gamma, Q} D\right)$.

Now we present the construction precisely. Let $T_{0}={ }^{(0,1,0)} T$. Since $T_{0}$ is the image of $T$ under the endomorphism corresponding to $(0,1,0)$, it is clearly a regular subsemigroup of $T$. Moreover $(0,1,0)$, the identity element of $G_{0}$, acts identically on $T_{0}$. Therefore the elements of $G_{0}$ act by automorphisms on $T_{0}$. Thus we can define an action $\gamma$ of the group $G$ on $T_{0}$ by putting ${ }^{b} a={ }^{\langle 0,8,0)} a$ for every $g \in G$ and 
$a \in T_{0}$. Later on, we shall use both actions - the action of $C$ on $T$ involved in the definition of $T *_{r} C$ and the action of $G$ on $T_{0}$ defined now by means of the former action-simultaneously.

For brevity, denote $T *_{r} C$ by $S$. We need the following notation. If $s=(t, c) \in S$ where $t \in T$ and $c=(i, g, \lambda) \in C$ then we denote $t$ by $t_{s}, c$ by $c_{s}, i$ by $s_{R}$ and $\lambda$ by $s_{L}$. Consider

$$
\hat{S}=\left\{\left(s, s^{\prime}\right) \in S \times S: s^{\prime} \in V(s)\right\},
$$

and define $D=\mathcal{M}(\hat{S}, G, \Lambda ; \hat{P})$ where $\hat{P}=\left(\hat{p}_{\lambda \hat{s}}\right)$ is the following $\Lambda \times \hat{S}$ matrix over $G$ : for any $\lambda \in \Lambda$ and $\hat{s} \in \hat{S}$ with $\hat{s}=\left(s, s^{\prime}\right)$, put $\hat{p}_{\lambda \hat{s}}=p_{\lambda i}$, where $i=s_{R}$. Clearly, $D$ is a subdirect product of $C$ and the left zero semigroup on the set $\hat{S}$.

For any $\hat{s}=\left(s, s^{\prime}\right) \in \hat{S}$, we have

$$
\left(t_{s}, c_{s}\right)\left(t_{s^{\prime}}, c_{s^{\prime}}\right)\left(t_{s}, c_{s}\right)=\left(t_{s}, c_{s}\right) \text { and }\left(t_{s^{\prime}}, c_{s^{\prime}}\right)\left(t_{s}, c_{s}\right)\left(t_{s^{\prime}}, c_{s^{\prime}}\right)=\left(t_{s^{\prime}}, c_{s^{\prime}}\right)
$$

in $S$. These two equalities are equivalent to the following: $c_{s^{\prime}} \in V\left(c_{s}\right)$ in $C$ and the equalities

$$
t_{s} \cdot{ }^{c^{\prime}} t_{s^{\prime}} \cdot c_{s} c_{s^{\prime}} t_{s}=t_{s} \quad \text { and } \quad t_{s^{\prime}} \cdot c_{s^{\prime}} t_{s} \cdot c_{s^{\prime}} c_{s} t_{s^{\prime}}=t_{s^{\prime}}
$$

are valid in $T$. In particular, these equalities imply that ${ }^{c_{s}} t_{s^{\prime}} \in V\left({ }^{c_{s}} c_{s^{\prime}} t_{s}\right)$. Conversely, Result 2.4 ensures that if $s=\left(t_{s}, c_{s}\right)$ and $e$ is an idempotent with $e \mathcal{R} c_{s}$ and ${ }^{~} t_{s} \geq_{\mathcal{L}} t_{s}$ then $s^{\prime}=\left({ }^{\prime} t^{\prime}, c^{\prime}\right)$, where $c^{\prime} \in V\left(c_{s}\right)$ with $c^{\prime} \mathcal{L} e$ and $t^{\prime} \in V\left(t_{s}\right)$, is an inverse of $s$.

Let us define $r_{\hat{s}}=t_{s} \cdot{ }^{c}{ }^{c} t_{s^{\prime}}$ for every $\hat{s}=\left(s, s^{\prime}\right)$ and $q_{\lambda \hat{s}}={ }^{(0,1, \lambda)} r_{\hat{s}}$ for every $\lambda \in \Lambda$ and $\hat{s} \in \hat{S}$. Obviously, $q_{\lambda \hat{s}} \in T_{0}$, and so $Q=\left(q_{\lambda \hat{s}}\right)$ is a $\Lambda \times \hat{S}$ matrix over $T_{0}$. Thus we have defined the regular Pastijn-Rees product $T_{0} \diamond_{r} D=T_{0} \diamond_{r}^{\gamma, Q} D$.

Let $\bar{T}$ be the left zero semigroup on the set $T$. Consider the following subset of the direct product $\bar{T} \times\left(T_{0} \diamond_{r} D\right)$ :

$$
\begin{aligned}
W= & \left\{[x,(\hat{s}, b, g, \lambda)] \in \bar{T} \times\left(T_{0} \diamond_{,} D\right): \text { for } \hat{s}=\left(s, s^{\prime}\right), i=s_{R},\right. \\
& \mu=s_{L}^{\prime} \text { and } a=x \cdot{ }^{(i, 1,0)} b, \text { the relations } b={ }^{\left(0, p_{\mu i}^{-1}, \mu\right)} a, \\
& \left.b \mathcal{L}^{p_{\mu i}^{-1}} q_{\mu \hat{s}} b \text { hold in } T \text { and } s \geq_{\mathcal{R}}(a ; i, g, \lambda) \text { in } S\right\} .
\end{aligned}
$$

Notice that, by Result $2.4,(a ; i, g, \lambda)$ belongs, indeed, to $S$ because

$$
{ }^{\left(i, p_{\mu i}^{-1}, \mu\right)} a={ }^{(i, 1,0)} b \geq_{\mathcal{L}} a .
$$

We intend to verify that $W$ is a regular subsemigroup of $\bar{T} \times\left(T_{0} \diamond, D\right)$ and $S$ is a homomorphic image of $W$.

First we verify that $W$ is a subsemigroup. Let $[x,(\hat{s}, b, g, \lambda)]$ and $[y,(\hat{u}, d, h, \xi)]$ be arbitrary elements of $W$. Then we have $\hat{s}=\left(s, s^{\prime}\right), i=s_{R}, \mu=s_{L}^{\prime}, a=x \cdot{ }^{(i, 1,0)} b$, 
$b={ }^{\left(0, p_{\mu i}^{-1}, \mu\right)} a, b \mathcal{L}^{p_{\mu i}^{-1}} q_{\mu \hat{s}} b$ and $s \geq_{\mathcal{R}}(a ; i, g, \lambda)$, and, moreover, we also have $\hat{u}=$ $\left(u, u^{\prime}\right), k=u_{R}, \eta=u_{L}^{\prime}, c=y \cdot{ }^{(k, 1,0)} d, d={ }^{\left(0, p_{\eta^{k}}^{-1}, \eta\right)} c, d \mathcal{L}^{p_{n k}^{-1}} q_{\eta \hat{u}} d$ and $u \geq_{\mathcal{R}}(c ; k, h, \xi)$. By definition,

$$
[x,(\hat{s}, b, g, \lambda)][y,(\hat{u}, d, h, \xi)]=\left[x,\left(\hat{s}, f, g p_{\lambda k} h, \xi\right)\right],
$$

where $f=b \cdot{ }^{g} q_{\lambda \hat{k}} \cdot{ }^{g p_{\lambda k}} d$. Put $e=x \cdot{ }^{(i, 1,0)} f$. Then

$$
\begin{aligned}
e & =x \cdot{ }^{(i, 1,0)}\left(b \cdot{ }^{8} q_{\lambda \hat{u}} \cdot{ }^{g p \lambda k} d\right) \\
& =x \cdot \cdot^{(i, 1,0)} b \cdot{ }^{(i, 1,0)(0, g, 0)(0,1, \lambda)} r_{\hat{u}} \cdot(i, 1,0)\left(0, g p_{\lambda k}, 0\right) d \\
& =a \cdot{ }^{(i, g, \lambda)} r_{\hat{u}} \cdot(i, g, \lambda)(k, 1,0) d=a \cdot{ }^{(i, g, \lambda)} r_{\hat{u}} \cdot(i, g, \lambda)\left(k, p_{\left.\eta k^{-1}, \eta\right)} c\right.
\end{aligned}
$$

and

$$
{ }^{\left(0, p_{\mu i}^{-1}, \mu\right)} e={ }^{\left(0, p_{\mu i}^{-1}, \mu\right)}\left(x \cdot{ }^{(i, 1,0)} f\right)={ }^{\left(0, p_{\mu i}^{-1}, \mu\right)} x \cdot{ }^{(0,1,0)} f={ }^{\left(0, p_{\mu i}^{-1}, \mu\right)} x \cdot f=f,
$$

since ${ }^{\left(0, p_{\mu i}^{-1}, \mu\right)} x \cdot b={ }^{\left(0, p_{\mu i}^{-1}, \mu\right)} x \cdot \cdot^{\left(0, p_{\mu i}^{-1}, \mu\right)(i, 1,0)} b={ }^{\left(0, p_{\mu i}^{-1}, \mu\right)}\left(x \cdot{ }^{(i, 1,0)} b\right)={ }^{\left(0, p_{\mu i}^{-1}, \mu\right)} a=b$. Since $\mathcal{L}$ is a right congruence, the relation $b \mathcal{L}^{{ }^{p_{\mu i}^{-1}}} q_{\mu \hat{s}} b$ implies $f \mathcal{L}^{p_{\mu i}^{-1}} q_{\mu \hat{s}} f$. To prove the relation $s \geq_{\mathcal{R}}\left(e ; i, g p_{\lambda k} h, \xi\right)$, it suffices to show that $(a ; i, g, \lambda) \geq_{\mathcal{R}}\left(e ; i, g p_{\lambda k} h, \xi\right)$. Result 2.4 implies by (2) that $(a ; i, g, \lambda)$ has an inverse in $S$ of the form

$$
\left({ }^{\left(j, p_{\lambda j}^{-1} g^{-1} p_{\mu i}^{-1}, \mu\right)} a^{\prime} ; j, p_{\lambda j}^{-1} g^{-1} p_{\mu i}^{-1}, \mu\right),
$$

where $a^{\prime} \in V(a)$. Now we see that

$$
\begin{aligned}
& (a ; i, g, \lambda)\left(\left(^{\left(j, p_{\lambda j}^{-1} g^{-1} p_{\mu i}^{-1}, \mu\right)} a^{\prime} ; j, p_{\lambda j}^{-1} g^{-1} p_{\mu i}^{-1}, \mu\right)\left(e ; i, g p_{\lambda k} h, \xi\right)\right. \\
& =\left(a \cdot \cdot^{\left(i, p_{\mu i}^{-1}, \mu\right)} a^{\prime} ; i, p_{\mu i}^{-1}, \mu\right)\left(a \cdot{ }^{(i, g, \lambda)} r_{\hat{u}} \cdot{ }^{(i, g, \lambda)\left(k, p_{n k}^{-1}, \eta\right)} c ; i, g p_{\lambda k} h, \xi\right) \text { by (3) } \\
& =\left(a \cdot{ }^{\left(i, p_{\mu l}^{-1}, \mu\right)}\left(a^{\prime} a\right) \cdot{ }^{(i, g, \lambda)} r_{\hat{u}} \cdot{ }^{(i, g, \lambda)\left(k, p_{n^{\prime}}^{-1}, \eta\right)} c ; i, g p_{\lambda k} h, \xi\right)
\end{aligned}
$$

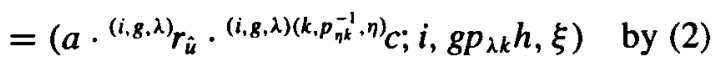

$$
\begin{aligned}
& =\left(e ; i, g p_{\lambda k} h, \xi\right) \text {. }
\end{aligned}
$$

Hence $\left(e ; i, g p_{\lambda k} h, \xi\right) \leq_{\mathcal{R}}(a ; i, g, \lambda)$ which was to be verified. Thus we have shown that $W$ is a subsemigroup.

Now we prove that $W$ is regular. Let $[\dot{x},(\hat{s}, b, g, \lambda)] \in W$ as before. Define $u^{\prime}=$ $(a ; i, g, \lambda)$, and let $u$ be the inverse $\left(^{\left(j, p_{\lambda j}^{-1} g^{-1} p_{\mu i}^{-1}, \mu\right)} a^{\prime} ; j, p_{\lambda j}^{-1} g^{-1} p_{\mu i}^{-1}, \mu\right)$ of $u^{\prime}$ considered above. Put $\hat{u}=\left(u, u^{\prime}\right)$. Moreover, let $d={ }^{p_{j i}^{-1} g^{-1}}\left(b^{\prime} b\left({ }^{p_{\mu i}^{-1}} q_{\mu \hat{s}} b\right)^{\prime}\right)$, where $b^{\prime}={ }^{\left(0, p_{\mu i}^{-1}, \mu\right)} a^{\prime}$, which is obviously an inverse of $b$, and $\left(^{p_{\mu i}^{-1}} q_{\mu \hat{s}} b\right)^{\prime} \in V\left(\left(_{\mu i}^{-1} q_{\mu \dot{s}} b\right)\right.$. Let us also define $c={ }^{(j, 1,0)} d$ and $y=c c^{\prime}$ for some $c^{\prime} \in V(c)$. By assumption, we have $b \mathcal{L}^{p_{\mu i}^{-1}} q_{\mu \hat{s}} b$. Furthermore, we see that

$$
\begin{aligned}
b \cdot{ }^{8} q_{\lambda \hat{u}} & ={ }^{\left(0, p_{\mu i}^{-1}, \mu\right)} a \cdot{ }^{(0, g, 0)(0,1, \lambda)} r_{\hat{u}} \\
& ={ }^{(0,1,0)}\left(^{\left(i, p_{\mu i}^{-1}, \mu\right)} a \cdot{ }^{(i, g, \lambda)}\left({ }^{\left(j, p_{\lambda j}^{-1} g^{-1} p_{\mu i}^{-1}, \mu\right)} a^{\prime} \cdot{ }^{\left(j, p_{\mu j}^{-1} g^{-1} p_{\mu i}^{-1}, \mu\right)} a\right)\right) \\
& ={ }^{(0,1,0)\left(i, p_{\mu i}^{-1}, \mu\right)}\left(a a^{\prime} a\right)={ }^{\left(0, p_{\mu i}^{-1}, \mu\right)} a=b .
\end{aligned}
$$


Hence Proposition 3.4(ii) ensures that $\left(\hat{u}, d, p_{\lambda j}^{-1} g^{-1} p_{\mu i}^{-1}, \mu\right)$ is an inverse of $(\hat{s}, b, g, \lambda)$ in $T_{0} \diamond_{r} D$. Therefore it remains to check that $\left[y,\left(\hat{u}, d, p_{\lambda j}^{-1} g^{-1} p_{\mu i}^{-1}, \mu\right)\right] \in W$. It is clear by the definition of $c$ and $y$ that $y \cdot{ }^{(j, 1,0)} d=c c^{\prime} c=c$ and ${ }^{\left(0, p_{j}^{-1}, \lambda\right)} c={ }^{\left(0, p_{j}^{-1}, \lambda\right)(j, 1,0)} d=$ ${ }^{(0,1,0)} d=d$. By (4) it follows that $b^{\prime} b \geq_{\mathcal{L}} b \cdot{ }^{8} q_{\lambda \hat{\mu}} b^{\prime} b=b b^{\prime} b=b \mathcal{L} b^{\prime} b$, whence

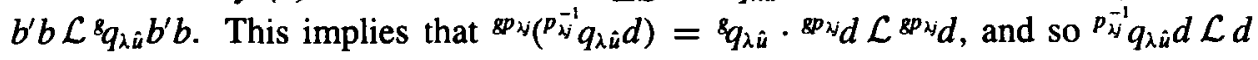
holds. Finally, by definition, we have

$$
\begin{aligned}
& c={ }^{(j, 1,0)} d={ }^{(j, 1,0)\left(0, p_{j}^{-1} g^{-1}, 0\right)}\left(b^{\prime} b\left(^{\left(P_{\mu i}^{-1}\right.} q_{\mu \bar{s}} b\right)^{\prime}\right) \\
& ={ }^{\left(j, p_{\mu}^{-1} g^{-1}, 0\right)}\left({ }^{\left(0, p_{\mu i}^{-1}, \mu\right)}\left(a^{\prime} a\right)\left({ }^{p_{\mu i}^{-1}} q_{\mu \hat{s}} b\right)^{\prime}\right) \\
& \left.={ }^{\left(j, p_{j}^{-1} g^{-1} p_{\mu i}^{-1}, \mu\right)}\left(a^{\prime} a\right) \cdot{ }^{\left(j, p_{j j}^{-1} g^{-1}, 0\right)\left(p_{\mu i}^{-1}\right.} q_{\mu \bar{s}} b\right)^{\prime},
\end{aligned}
$$

which implies $c={ }^{\left(j, p_{j j}^{-1} g^{-1} p_{\mu i}^{-1}, \mu\right)}\left(a^{\prime} a\right) c=^{\left(j, p_{j j}^{-1} g^{-1} p_{\mu i}^{-1}, \mu\right)}\left(a^{\prime} a\right) \cdot{ }^{\left(j, p_{j j}^{-1}, \lambda\right)} c$. Hence we see that

$$
\begin{aligned}
u u^{\prime} & \left(c ; j, p_{\lambda j}^{-1} g^{-1} p_{\mu i}^{-1}, \mu\right) \\
& =\left({ }^{\left(j, p_{j}^{-1} g^{-1} p_{\mu i}^{-1}, \mu\right)} a^{\prime} ; j, p_{\lambda j}^{-1} g^{-1} p_{\mu i}^{-1}, \mu\right)(a ; i, g, \lambda)\left(c ; j, p_{\lambda j}^{-1} g^{-1} p_{\mu i}^{-1}, \mu\right) \\
& =\left({ }^{\left(j, p_{j}^{-1} g^{-1} p_{\mu i}^{-1}, \mu\right)}\left(a^{\prime} a\right) ; j, p_{\lambda j}^{-1}, \lambda\right)\left(c ; j, p_{\lambda j}^{-1} g^{-1} p_{\mu i}^{-1}, \mu\right) \\
& =\left(c ; j, p_{\lambda j}^{-1} g^{-1} p_{\mu i}^{-1}, \mu\right)
\end{aligned}
$$

and so $\left(c ; j, p_{\lambda j}^{-1} g^{-1} p_{\mu i}^{-1}, \mu\right) \leq_{\mathcal{R}} u$ follows. Thus $\left[y,\left(\hat{u}, d, p_{\lambda j}^{-1} g^{-1} p_{\mu i}^{-1}, \mu\right)\right] \in W$ is proved and $W$ is, indeed, regular.

Now we define a mapping $\Phi: W \rightarrow S$ by $[x,(\hat{s}, b, g, \lambda)] \Phi=(a ; i, g, \lambda)$, where $i=s_{R}$ and $a=x \cdot{ }^{(i, 1,0)} b$. We have seen after the definition of $W$ that $(a ; i, g, \lambda) \in S$. We intend to show that $\Phi$ is a surjective homomorphism. Let

$$
[x,(\hat{s}, b, g, \lambda)],[y,(\hat{u}, d, h, \xi)] \in W
$$

as before. When proving that $W$ is a subsemigroup, we have seen that

$$
[x,(\hat{s}, b, g, \lambda)][y,(\hat{u}, d, h, \xi)]=\left[x,\left(\hat{s}, f, g p_{\lambda k} h, \xi\right)\right],
$$

where $f=b \cdot q_{q_{\hat{\mu}}} \cdot g^{g p_{\lambda k}} d$ and, by (3), $e=x \cdot{ }^{(i, 1,0)} f=a \cdot{ }^{(i, g, \lambda)} r_{\hat{u}} \cdot{ }^{(i, g, \lambda)\left(k, p_{n k}^{-1}, \eta\right)} c$. Thus, by the definition of $\Phi$,

$$
([x,(\hat{s}, b, g, \lambda)][y,(\hat{u}, d, h, \xi)]) \Phi=\left(a \cdot{ }^{(i, g, \lambda)} r_{\hat{u}} \cdot{ }^{\left.(i, g, \lambda)(k, p) \eta_{k}^{-1}, \eta\right)} c ; i, g p_{\lambda k} h, \xi\right) .
$$

On the other hand, we have

$$
\begin{aligned}
{[x,(\hat{s}, b, g, \lambda)] \Phi \cdot[y,(\hat{u}, d, h, \xi)] \Phi } & =(a ; i, g, \lambda)(c ; k, h, \xi) \\
& =\left(a \cdot{ }^{(i, g, \lambda)} c ; i, g p_{\lambda k} h, \xi\right)
\end{aligned}
$$


Therefore, in order to show that $\Phi$ is a homomorphism, it suffices to verify that $c=r_{\hat{u}} \cdot{ }^{\left(k, p_{\eta}^{-1}, \eta\right)} c$. Since $u \geq_{\mathcal{R}}(c ; k, h, \xi)$, we have $(c ; k, h, \xi)=u x=\left(t_{u}, c_{u}\right)\left(t_{x}, c_{x}\right)$ for some $x \in S$, whence $c=t_{u} \cdot{ }^{c_{u}} t_{x}$ follows. Since $u^{\prime} \in V(u)$, (1) implies that

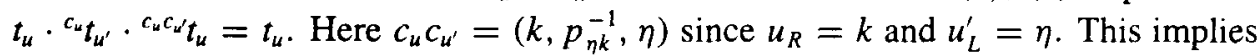
that $r_{\hat{u}} \cdot{ }^{\left(k, p_{\eta^{k}}^{-1}, \eta\right)} t_{u}=t_{u}$, and so $r_{\hat{u}} \cdot{ }^{\left(k, p_{\eta k}^{-1}, \eta\right)} t_{u} \cdot c t_{x}=t_{u} \cdot{ }^{c_{u}} t_{x}$. However, $c=t_{u} \cdot{ }^{{ }^{u} t_{x}}$, and since $\left(k, p_{\eta k}^{-1}, \eta\right) c_{u}=c_{u}$, we also have ${ }^{\left(k, p_{\eta k}^{-1}, \eta\right)} t_{u} \cdot c_{u} t_{x}={ }^{\left(k, p_{\eta^{k}}, \eta\right)}\left(t_{u} \cdot{ }^{{ }^{\prime}} t_{x}\right)={ }^{\left(k, p_{\eta k}^{-1}, \eta\right)} c$. Thus $r_{\hat{u}} \cdot{ }^{\left(k, p_{n k}^{-1}, \eta\right)} c=c$ holds, and $\Phi$ is, indeed, a homomorphism.

Finally, we show that $\Phi$ is surjective. Let $s=(a ; i, g, \lambda) \in S$. Since it is regular, by Result 2.4 there is an idempotent $\left(i, p_{\mu i}^{-1}, \mu\right) \in C$ such that ${ }^{\left(i, p_{\mu i}^{-1}, \mu\right)} a \geq_{\mathcal{L}} a$ and an inverse of $s$ of the form $s^{\prime}=\left({ }^{\left(j, p_{\lambda j}^{-1} g^{-1} p_{\mu i}^{-1}, \mu\right)} a^{\prime} ; j, p_{\lambda j}^{-1} g^{-1} p_{\mu i}^{-1}, \mu\right)$, where $j \in I$ and $a^{\prime} \in V(a)$. Put $\hat{s}=\left(s, s^{\prime}\right), b={ }^{\left(0, p_{\mu t}^{-1}, \mu\right)} a$ and $x=r_{\hat{s}}$. Then, by the definition of $r_{\hat{s}}$, we see that

$$
x \cdot{ }^{(i, 1,0)} b=r_{\hat{s}} \cdot{ }^{\left(i, p_{\mu i}^{-1}, \mu\right)} a=a \cdot{ }^{(i, g, \lambda)}\left(\left(^{\left(j, p_{\lambda j}^{-1} g^{-1} p_{\mu i}^{-1}, \mu\right)} a^{\prime}\right) \cdot{ }^{\left(i, p_{\mu i}^{-1}, \mu\right)} a=a \cdot{ }^{\left(i, p_{\mu i}^{-1}, \mu\right)}\left(a^{\prime} a\right)=a .\right.
$$

Hence it follows that

$$
{ }^{p_{\mu i}^{-1}} q_{\mu \hat{s}} b={ }^{\left(0, p_{\mu i}^{-1}, \mu\right)} r_{r_{s}} \cdot{ }^{\left(0, p_{\mu i}^{-1}, \mu\right)} a={ }^{\left(0, p_{\mu i}^{-1}, \mu\right)}\left(r_{\hat{s}} \cdot{ }^{\left(i, p_{\mu i}^{-1}, \mu\right)} a\right)={ }^{\left(0, p_{\mu i}^{-1}, \mu\right)} a=b .
$$

Thus $[x,(\hat{s}, b, g, \lambda)] \in W$ and $[x,(\hat{s}, b, g, \lambda)] \Phi=(a ; i, g, \lambda)$. This completes the proof of the proposition.

If $\mathcal{U}$ is any e-variety [pseudo-e-variety] and $\mathcal{V}$ is a variety [pseudo-e-variety] of completely simple semigroups, then we define the regular Pastijn-Rees product $\mathcal{U} \diamond, \mathcal{V}$ of $\mathcal{U}$ by $\mathcal{V}$ to be the e-variety [pseudo-e-variety] generated by the class of all regular Pastijn-Rees products $T \diamond, C$ where $T \in \mathcal{U}$ and $C \in \mathcal{V}$.

Combining Proposition 3.7, Remark 3.1 and Corollary 3.6, Proposition 3.8, respectively, we obtain the following result.

THEOREM 3.9. Let $\mathcal{U}$ be any e-variety [pseudo-e-variety], and let $\mathcal{V}$ be either a variety [pseudo-e-variety] of groups or of completely simple semigroups with $\mathcal{L} \subseteq \mathcal{V}$. Then we have $\mathcal{U} *_{r} \mathcal{V}=\mathcal{U} \diamond, \mathcal{V}$.

Notice that if $\mathcal{V} \nsubseteq \mathcal{G}$ and $\mathcal{L Z} \nsubseteq \mathcal{V}$ then the equality $\mathcal{U} *_{r} \mathcal{V}=\mathcal{U} \diamond_{r} \mathcal{V}$ does not hold in general. For example, $\mathcal{G} *_{r} \mathcal{R Z}=\mathcal{C S}$, see [9], but $\mathcal{G} \diamond_{r} \mathcal{R Z}=\mathcal{G} \vee \mathcal{R Z}$. For, it is easy to check that, for any Pastijn-Rees product of a group $G$ by a right zero semigroup $\Lambda$, we have $G \diamond_{r} \Lambda=G \diamond \Lambda$, and the mapping $G \diamond \Lambda \rightarrow G \times \Lambda$, $(g, \lambda) \mapsto\left(g q_{\lambda}, \lambda\right)$ is an isomorphism into the direct product of $G$ and $\Lambda$.

By applying the main result in [4], we can deduce the following result which handles the case not covered by Theorem 3.9 .

THEOREM 3.10. Let $\mathcal{U}$ be any e-variety [pseudo-e-variety], and let $\mathcal{V}$ be a variety [pseudo-e-variety] of right groups such that $\mathcal{V} \nsubseteq \mathcal{G}$. Then we have $\mathcal{U} *_{r} \mathcal{V}=\mathcal{V}=$ $\mathcal{U} \diamond_{r} \mathcal{V}$ if $\mathcal{U} \subseteq \mathcal{R Z}$ and $\mathcal{U} *_{r} \mathcal{V}=\mathcal{U} \diamond_{r}(\mathcal{L} \mathcal{Z} \vee)$ otherwise. 
PRoof. Suppose first that $\mathcal{U} \subseteq \mathcal{R Z}$. Then $\mathcal{U} *_{r} \mathcal{V}=\mathcal{U} \vee \mathcal{V}=\mathcal{V}$ by [4, Proposition 5.1]. By Corollary 3.6, we have $\mathcal{U} \diamond_{r} \mathcal{V} \subseteq \mathcal{U} *_{r} \mathcal{V}$. Since the inclusion $\mathcal{V} \subseteq \mathcal{U} \diamond_{r} \mathcal{V}$ is clear, the equalities $\mathcal{U} *_{r} \mathcal{V}=\mathcal{V}=\mathcal{U} \diamond_{r} \mathcal{V}$ follow.

Now assume that $\mathcal{U} \nsubseteq \mathcal{R Z}$. Theorem 3.9 implies that $\mathcal{U} *_{r}(\mathcal{L Z} \vee \mathcal{V})=\mathcal{U} \diamond_{r}$ $(\mathcal{L Z} \vee \mathcal{V})$. By [4, Proposition 5.2 and Theorem 5.1], we see that $\mathcal{U} *_{r}(\mathcal{L} \mathcal{Z} \vee \mathcal{V})=$ $\left(\mathcal{U} *_{r} \mathcal{V}\right) *_{r} \mathcal{L} \mathcal{Z}=\left(\mathcal{U} *_{r} \mathcal{V}\right) \vee \mathcal{L Z}$. However, [9, Lemma 4.2] ensures that $\mathcal{L} \mathcal{Z} \subseteq \mathcal{U} *_{r} \mathcal{V}$, and so $\left(\mathcal{U} *_{r} \mathcal{V}\right) \vee \mathcal{L Z}=\mathcal{U} *_{r} \mathcal{V}$, which completes the proof.

\section{Bifree objects in e-varieties which are regular semidirect products of inverse semigroup varieties by completely simple semigroup varieties}

Given a variety $\mathcal{U}$ of inverse semigroups and a variety $\mathcal{V}$ of completely simple semigroups with $\mathcal{R B} \subseteq \mathcal{V}$, the main result Theorem 3.9 of the previous section allows us to construct a model of any bifree object in the e-variety $\mathcal{U} *_{r} \mathcal{V}$ by making use of the Pastijn-Rees product construction. Similarly to the models of the bifree objects found in [10], these models have a graphical interpretation which makes them more transparent than the models presented in [9, Section 8].

It is known from [9] that, for any inverse semigroup $T$ and completely simple semigroup $C$, every regular semidirect product of $T$ by $C$ is locally inverse. By Proposition 3.5, each regular Pastijn-Rees product of $T$ by $C$ is also locally inverse. Therefore $\mathcal{U} *_{r} \mathcal{V}, \mathcal{U} \diamond, \mathcal{V} \subseteq \mathcal{L} \mathcal{I}$ provided $\mathcal{U} \subseteq \mathcal{I}$ and $\mathcal{V} \subseteq \mathcal{C S}$.

In case $T$ is an inverse semigroup, Proposition 3.4(ii) can be strengthened as follows.

Proposition 4.1. Let $T \diamond C=T \diamond^{\gamma, Q} C$ be a Pastijn-Rees product of an inverse semigroup $T$ by a completely simple semigroup $C=\mathcal{M}(I, G, \Lambda ; P)$. Two elements $(i, a, g, \lambda)$ and $(j, b, h, \mu)$ are mutually inverse in $T \diamond C$ if and only if $h=p_{\lambda j}^{-1} g^{-1} p_{\mu i}^{-1}$, $a=a \cdot{ }^{g}\left(q_{\lambda j} q_{\lambda j}^{-1}\right)={ }^{p_{\mu i}^{-1}}\left(q_{\mu i}^{-1} q_{\mu i}\right) a$ and $b={ }^{p_{\lambda j}^{-1}} q_{\lambda j}^{-1} \cdot p_{\lambda j}^{-1} g^{-1} a^{-1} \cdot p_{\lambda j}^{-1} g^{-1} p_{\mu i}^{-1} q_{\mu i}^{-1}$.

ProOF. Suppose first that $(j, b, h, \mu) \in V((i, a, g, \lambda))$. Then an easy calculation gives $h=p_{\lambda j}^{-1} g^{-1} p_{\mu i}^{-1}$ and, by Proposition 3.4 (ii), $a \cdot{ }^{8} q_{\lambda j} \mathcal{R} a$ and ${ }^{p_{\mu i}^{-1}} q_{\mu i} a \mathcal{L} a$. The relation $a \cdot{ }^{8} q_{\lambda j} \mathcal{R} a$ implies $a \cdot 8\left(q_{\lambda j} q_{\lambda j}^{-1}\right) a^{-1}=a a^{-1}$ whence $a=a a^{-1} a=a \cdot{ }^{8}\left(q_{\lambda j} q_{\lambda j}^{-1}\right)$. $a^{-1} a=a \cdot{ }^{8}\left(q_{\lambda j} q_{\lambda j}^{-1}\right)$. Similarly, we obtain from ${ }^{p_{\mu i}^{-1}} q_{\mu i} a \mathcal{L} a$ that ${ }^{p_{\mu i}^{-1}}\left(q_{\mu i}^{-1} q_{\mu i}\right) a=a$. Moreover, a straightforward calculation shows that

$$
\begin{aligned}
& a=a \cdot{ }^{8} q_{\lambda j} \cdot{ }^{g p_{\lambda j}} b \cdot{ }^{p_{\mu i}^{-1}} q_{\mu i} a \text { and } \\
& b=b \cdot{ }^{-1} g^{-1} p_{\mu i}^{-1} q_{\mu i} \cdot p_{\mu j}^{-1} g^{-1} a \cdot{ }^{p_{\lambda j}^{-1}} q_{\lambda j} b .
\end{aligned}
$$

From (5), we deduce that $p_{\lambda j}^{-1} g^{-1} a=p_{\lambda j}^{-1} g^{-1} a \cdot p_{\lambda j}^{-1} q_{\lambda j} \cdot b \cdot p_{\lambda j}^{-1} g^{-1} p_{\mu i}^{-1} q_{\mu i} \cdot p_{\lambda j}^{-1} g^{-1} a$, and so

$$
p_{\lambda j}^{-1} g^{-1} p_{\mu i}^{-1} q_{\mu i} \cdot p_{\lambda i}^{-1} g^{-1} a \cdot p_{\lambda j}^{-1} q_{\lambda j}=p_{\lambda j}^{-1} g^{-1} p_{\mu i}^{-1} q_{\mu i} \cdot p_{\lambda j}^{-1} g^{-1} a \cdot p_{\lambda j}^{-1} q_{\lambda j} b \cdot p_{\lambda j}^{-1} g^{-1} p_{\mu i}^{-1} q_{\mu i} \cdot p_{\lambda j}^{-1} g^{-1} a \cdot p_{\lambda j}^{-1} q_{\lambda j} .
$$


Taking into account (6), this implies that

$$
b=\left(p_{\lambda}^{-1} g^{-1} p_{\mu i}^{-1} q_{\mu i} \cdot p_{\lambda j}^{-1} g^{-1} a \cdot p_{\lambda j}^{-1} q_{\lambda j}\right)^{-1}={ }^{-1} q_{\lambda j}^{-1} \cdot p_{\lambda j}^{-1} g^{-1} a^{-1} \cdot p_{\lambda j}^{-1} g^{-1} p_{\mu i}^{-1} q_{\mu i}^{-1} .
$$

The if part follows from the given conditions and the last statement in Proposition 3.4 (ii) if we observe that

$$
\begin{aligned}
p_{\lambda j}^{-1} g^{-1}\left(\left(a \cdot{ }^{8} q_{\lambda j}\right)^{-1} a\left({ }^{p_{\mu i}^{-1}} q_{\mu i} a\right)^{-1}\right) & ={ }^{p_{\lambda j}^{-1} g^{-1}}\left({ }^{8} q_{\lambda j}^{-1} a^{-1} a a^{-1} \cdot p_{\mu i}^{-1} q_{\mu i}^{-1}\right) \\
& ={ }^{p_{\lambda j}^{-1}} q_{\lambda j}^{-1} \cdot p_{\lambda j}^{-1} g^{-1} a^{-1} \cdot p_{\lambda j}^{-1} g^{-1} p_{\mu i}^{-1} q_{\mu i}^{-1}=b .
\end{aligned}
$$

The proof is complete.

The next lemma provides a formula for the sandwich operation in a regular PastijnRees product of an inverse semigroup by a completely simple semigroup.

LEMMA 4.2. Let $T \diamond_{r} C=T \diamond_{r}^{r, Q} C$ be a regular Pastijn-Rees product of an inverse semigroup $T$ by a completely simple semigroup $C=\mathcal{M}(I, G, \Lambda ; P)$. Then, for any elements $(i, a, g, \lambda),(j, b, h, \mu) \in T \diamond, C$, we have

$$
(i, a, g, \lambda) \wedge(j, b, h, \mu)=\left(i, a a^{-1} \cdot p_{\mu i}^{-1} q_{\mu i}^{-1} \cdot p_{\mu i}^{-1} h^{-1}\left(b^{-1} b\right), p_{\mu i}^{-1}, \mu\right) .
$$

ProOF. For brevity, put $t=a a^{-1} \cdot p_{\mu i}^{-1} q_{\mu i}^{-1} \cdot p_{\mu i}^{-1} h^{-1}\left(b^{-1} b\right), u=(i, a, g, \lambda), v=$ $(j, b, h, \mu)$ and $w=\left(i, t, p_{\mu i}^{-1}, \mu\right)$. We check that $w$ belongs to the sandwich set of $v$ and $u$. First we notice that $t=e s^{-1} f$ where $e=a a^{-1}, s={ }^{p_{\mu i}^{-1}} q_{\mu i}$ and $f={ }^{p_{\mu i}^{-1} h^{-1}}\left(b^{-1} b\right)$. Obviously, $e, f \in E(T)$. Therefore

$$
t \cdot{ }^{p_{\mu i}^{-1}} q_{\mu i} t=e s^{-1} f \operatorname{ses}^{-1} f=e^{2} s^{-1} f s s^{-1} f=e s^{-1} s s^{-1} f^{2}=e s^{-1} f=t .
$$

This implies by Proposition 3.4(i) that $w$ is an idempotent. Now let us choose an inverse $u^{\prime}$ of $u$. By Proposition 4.1, we have

$$
u^{\prime}=\left(k, p_{\lambda k}^{-1} q_{\lambda k}^{-1} \cdot p_{\lambda k}^{-1} g^{-1} a^{-1} \cdot p_{\lambda k}^{-1} g^{-1} p_{k i}^{-1} q_{k i}^{-1}, p_{\lambda k}^{-1} g^{-1} p_{\kappa i}^{-1}, \kappa\right),
$$

where $k \in I$ and $\kappa \in \Lambda$ such that

$$
a=a \cdot{ }^{g}\left(q_{\lambda k} q_{\lambda k}^{-1}\right)={ }^{p_{k i}^{-1}}\left(q_{k i}^{-1} q_{k i}\right) a .
$$

Then a straightforward calculation shows that $u u^{\prime} w=w$ : equality of the components in $C$ is obvious; for the component in $T$ of the left hand side, we see by (7) that

$$
\begin{aligned}
a \cdot{ }^{8} q_{\lambda k} \cdot{ }^{g} q_{\lambda k}^{-1} a^{-1} \cdot{ }^{p_{k i}^{-1}} q_{k i}^{-1} \cdot p_{k i}^{-1} q_{k i} t \\
\quad=a \cdot{ }^{g} q_{\lambda k} \cdot{ }^{8} q_{\lambda k}^{-1} a^{-1} \cdot p_{k i}^{-1} q_{k i}^{-1} \cdot p_{k i}^{-1} q_{k i} \cdot a a^{-1} \cdot p_{\mu i}^{-1} q_{\mu i}^{-1} \cdot p_{\mu i}^{-1} h^{-1}\left(b^{-1} b\right) \\
\quad=a a^{-1} a a^{-1} \cdot p_{\mu i}^{-1} q_{\mu i}^{-1} \cdot p_{\mu i}^{-1} h^{-1}\left(b^{-1} b\right)=t
\end{aligned}
$$


Therefore we obtain that $w \leq_{\mathcal{R}} u$. Routine checking similarly gives $w \leq_{\mathcal{L}} v$. Finally, we can check that $v w u=v u$. Again it is enough to show the equality of the components in $T$. On the left hand side, we have

$$
\begin{aligned}
& b \cdot{ }^{h} q_{\mu i} \cdot{ }^{h p_{\mu i}}\left(a a^{-1}\right) \cdot{ }^{h} q_{\mu i}^{-1} \cdot b-1 b \cdot{ }^{h} q_{\mu i} \cdot{ }^{h p_{\mu i}} a \\
& =b \cdot{ }^{h} q_{\mu i} \cdot{ }^{h} q_{\mu i}^{-1} \cdot b^{-1} b \cdot{ }^{h} q_{\mu i} \cdot{ }^{h p_{\mu i}}\left(a a^{-1}\right) \cdot{ }^{h p_{\mu i} a} \\
& =b b^{-1} b \cdot{ }^{h} q_{\mu i}{ }^{h} q_{\mu i}^{-1} \cdot{ }^{h}{ }^{h}{ }_{\mu i} \cdot{ }^{h p_{\mu i}} a=b \cdot{ }^{h} q_{\mu i} \cdot{ }^{h p_{\mu i}} a,
\end{aligned}
$$

which is just the component in $T$ of the right hand side. The proof is complete.

Now let $\mathcal{U}$ be a variety of inverse semigroups and $\mathcal{V}$ a variety of completely simple semigroups with $\mathcal{R B} \subseteq \mathcal{V}$. Let $X$ be a non-empty set. Consider the bifree object $C=B F \mathcal{V}(X)$ presented in Section 2 . Now let $Q$ be an $\bar{X} \times \bar{X}$ matrix such that $q_{y z} \neq q_{u \nu}$ provided $(y, z) \neq(u, v)$ in $\bar{X} \times \bar{X}$, and put $\hat{Q}=\left\{q_{y z}: y, z \in \bar{X}\right\}$. Suppose also that $X \cap \hat{Q}=\emptyset$. Define $A=G_{\mathcal{V}} \times(X \cup \hat{Q})$, and let us 'double' it in the usual way. Put $\bar{A}=A \cup A^{-1}$, where $A^{-1}$ is disjoint from $A$ and a bijection ${ }^{-1}: A \rightarrow A^{-1}$, $a \mapsto a^{-1}$ is fixed. Consider the free object $F \mathcal{U}(A)=\mathbf{I}(A) / \rho_{\mathcal{U}}$, and, for brevity, denote it by $T$.

The group $G_{\mathcal{V}}$ acts on $A$ by ${ }^{h}(g, \ell)=(h g, \ell), h \in G_{\mathcal{V}},(g, \ell) \in A$, and this action can be extended to an action of $G_{\mathcal{V}}$ on $T$ in the usual way. Now we define an $\bar{X} \times \bar{X}$ matrix $Q_{A}=\left(\left(q_{A}\right)_{y z}\right)$ over $T$ by $\left(q_{A}\right)_{y z}=\left(1, q_{y z}\right)((y, z) \in \bar{X} \times \bar{X})$. The action of $G_{\mathcal{V}}$ on $T$ and the sandwich matrix $Q_{A}$ define a Pastijn-Rees product $T \diamond C(=F \mathcal{U}(A) \diamond B F \mathcal{V}(X))$ and a regular Pastijn-Rees product $T \diamond, C$. Consider the following elements in $T \diamond C$ : for any $x \in X$, let

$$
\tilde{x}=\left(r_{x} \rho_{\mathcal{U}} ; c_{x}\right) \text { and } \tilde{x}^{\prime}=\left(r_{x^{\prime}} \rho_{\mathcal{U}} ; c_{x^{\prime}}\right) \text {, }
$$

where

$$
\begin{aligned}
& r_{x}=(1, x)\left(x, q_{x x^{\prime}}\right)\left(x, q_{x x^{\prime}}\right)^{-1}(1, x)^{-1}\left(p_{x^{\prime} x}^{-1}, q_{x^{\prime} x}\right)^{-1}\left(p_{x^{\prime} x}^{-1}, q_{x^{\prime} x}\right)(1, x), \\
& r_{x^{\prime}}=\left(p_{x x^{\prime}}^{-1}, q_{x x^{\prime}}\right)^{-1}\left(p_{x x^{\prime}}^{-1} x^{-1}, x\right)^{-1}\left(p_{x x^{\prime}}^{-1} x^{-1} p_{x^{\prime} x}^{-1}, q_{x^{\prime} x}\right)^{-1} .
\end{aligned}
$$

We now check by applying Proposition 4.1 that $\tilde{x}^{\prime} \in V(\tilde{x})$. The equality

$$
r_{x} \rho_{\mathcal{U}}=r_{x} \rho_{\mathcal{U}} \cdot{ }^{x}\left(\left(1, q_{x x^{\prime}}\right) \rho_{\mathcal{U}}\left(\left(1, q_{x x^{\prime}}\right) \rho_{\mathcal{U}}\right)^{-1}\right)
$$

holds, since idempotents commute in an inverse semigroup. Similarly, we can see that

$$
r_{x} \rho_{\mathcal{U}}={ }_{x^{\prime} x}^{-1}\left(\left(\left(1, q_{x^{\prime} x}\right) \rho_{\mathcal{U}}\right)^{-1}\left(1, q_{x^{\prime} x}\right) \rho_{\mathcal{U}}\right) r_{x} \rho_{\mathcal{U}} \text {. }
$$

Finally, we have, by the definitions and routine cancellation in an inverse semigroup,

$$
p_{x x^{\prime}}^{-1}\left(\left(1, q_{x x^{\prime}}\right) \rho_{\mathcal{U}}\right)^{-1} \cdot p_{x x^{\prime}}^{-1} x^{-1}\left(r_{x} \rho_{\mathcal{U}}\right)^{-1} \cdot p_{x^{\prime}}^{-1} x^{-1} p_{x^{\prime} x}^{-1}\left(\left(1, q_{x^{\prime} x}\right) \rho_{\mathcal{U}}\right)^{-1}=r_{x^{\prime}} \rho_{\mathcal{U}} \text {. }
$$

This verifies, by Proposition 4.1, that $\tilde{x}^{\prime} \in V(\tilde{x})$. Hence $\tilde{x}, \tilde{x}^{\prime} \in T \diamond_{r} C$ for every $x \in X$. 
THEOREM 4.3. Let $\mathcal{U}$ be $a$ variety of inverse semigroups and $\mathcal{V}$ a variety of completely simple semigroups with $\mathcal{R B} \subseteq \mathcal{V}$. For any non-empty set $X$, consider the regular Pastijn-Rees product $F \mathcal{U}(A) \diamond, B F \mathcal{V}(X)$ defined above. Then the regular subsemigroup in $F \mathcal{U}(A) \diamond, B F \mathcal{V}(X)$ generated by the set $\left\{\tilde{x}, \tilde{x}^{\prime}: x \in X\right\}$ is the bifree object in $\mathcal{U} \odot, \mathcal{V}$ on the set $X$.

PROOF. We shall use the notation introduced before the theorem. Since $\tilde{x}$ and $\tilde{x}^{\prime}$ are inverses of each other in the locally inverse semigroup $T \diamond_{r} C$, the regular subsemigroup $W$ generated by $\left\{\tilde{x}, \tilde{x}^{\prime}: x \in X\right\}$ is well defined, and the mapping $\bar{X} \rightarrow T \diamond_{r} C, x \mapsto \tilde{x}, x^{\prime} \mapsto \tilde{x}^{\prime}(x \in X)$ is matched. By definition, the e-variety $\mathcal{U} \diamond_{r} \mathcal{V}$ is generated by the class $\mathcal{C}$ of all regular Pastijn-Rees products $U \diamond_{r} D$ where $U \in \mathcal{U}$ and $D \in \mathcal{V}$. It is routine to see that $\mathcal{C}$ is closed under forming direct products. Thus, by [9, Lemma 7.2], it suffices to prove that each matched mapping into a member of $\mathcal{C}$ can be extended to a homomorphism of $W$.

Consider a member $U \diamond_{r} D \in \mathcal{C}$, where $D=\mathcal{M}(I, H, \Lambda ; R)$ and the regular Pastijn-Rees product is defined by means of an action of $H$ on $U$ and a $\Lambda \times I$ sandwich matrix $S=\left(s_{\lambda i}\right)$ over $U$. Furthermore, let $\bar{X} \rightarrow U \diamond_{r} D, y \mapsto\left(u_{y} ; d_{y}\right)$ $(y \in \bar{X})$ be a matched mapping where $d_{y}=\left(i_{y}, h_{y}, \lambda_{y}\right)$. By Proposition 4.1, we have

$$
\begin{aligned}
& u_{x}=u_{x} \cdot{ }^{h_{x}}\left(s_{\lambda_{x} i_{x^{\prime}}} s_{\lambda_{x} i_{x^{\prime}}}^{-1}\right), \\
& u_{x}={ }_{\lambda_{x^{\prime} x_{x}}^{-1}}^{r^{-1}}\left(s_{\lambda_{x^{\prime}} i_{x}}^{-1} s_{\lambda_{x^{\prime}} i_{x}}\right) \cdot u_{x} \text {, }
\end{aligned}
$$

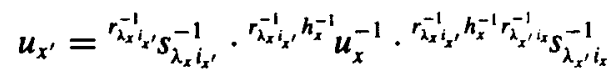

in $U$. In particular, the mapping $\bar{X} \rightarrow D, y \mapsto d_{y}(y \in \bar{X})$ is matched, and therefore it can be uniquely extended to a homomorphism $\psi: C \rightarrow D$.

Due to Proposition 3.2, we can assume without loss of generality that the Rees matrix representation $\mathcal{M}(I, H, \Lambda ; R)$ of $D$ is chosen in the way that $R$ is normalized at $\left(i_{x_{0}}, \lambda_{x_{0}}\right)$. In this case, there exists a group homomorphism $\gamma: G_{\mathcal{V}} \rightarrow H$ such that $p_{y z} \gamma=r_{\lambda_{y} i_{z}}(y, z \in \bar{X})$ and $(y, g, z) \psi=\left(i_{y}, g \gamma, \lambda_{z}\right)$ for every $(y, g, z) \in C$.

Now let us define a mapping $A \rightarrow U$ in the following manner:

$$
(g, x) \mapsto{ }^{g \gamma} u_{x},\left(g, q_{y z}\right) \mapsto{ }^{g \gamma_{\lambda_{y} i_{z}}} \quad\left(g \in G_{\mathcal{V}}, x \in X, q_{y z} \in \hat{Q}\right) .
$$

This mapping can be uniquely extended to an inverse semigroup homomorphism $\phi: T \rightarrow U$. In particular, we have $\left(1, q_{y z}\right) \phi=s_{\lambda_{y} i_{z}}$ for every $y, z \in \bar{X}$, and $\left({ }^{8} t\right) \phi=$ ${ }^{g \gamma} t \phi$ for every $g \in G_{\mathcal{V}}$ and $t \in T$. By means of these properties, it is straightforward to see that the mapping $\phi \times \psi: T \diamond_{r} C \rightarrow U \diamond_{r} D,(t ; c)(\phi \times \psi)=(t \phi ; c \psi)$ is a homomorphism. In particular, we have

$$
\begin{aligned}
\tilde{x}(\phi \times \psi) & =\left(u_{x} \cdot{ }^{h_{x}}\left(s_{\lambda_{x} i_{x^{\prime}}} s_{\lambda_{x} i_{x^{\prime}}}^{-1}\right) u_{x}^{-1} \cdot{ }^{r_{x^{\prime}}^{\prime} i_{x}}\left(s_{\lambda_{x^{\prime}} i_{x}}^{-1} s_{\lambda_{x^{\prime}} i_{x}}\right) u_{x} ; d_{x}\right) \\
& =\left(u_{x} u_{x}^{-1} u_{x} ; d_{x}\right)=\left(u_{x} ; d_{x}\right)
\end{aligned}
$$


by (8) and (9), and

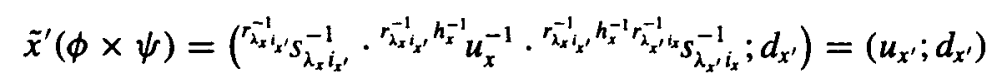

by (10), that is, $\phi \times \psi$ extends the matched mapping $\bar{X} \rightarrow U \diamond_{r} D$ we started with. This completes the proof.

The bifree object obtained in this proposition has a transparent graphical interpretation. Consider the directed graph $\mathcal{X}$ with set of vertices $G_{\mathcal{V}}$ and set of edges $A$. For any edge $(g, \ell) \in A$, its initial vertex is $g$ and its terminal vertex is $g \ell$ provided $\ell \in X$ and is $g p_{u v}$ provided $\ell=q_{u v} \in \hat{Q}$. We term $\ell$ the label of the edge $(g, \ell)$. When 'doubling' $A$, it is convenient to choose $A^{-1}$ in a special form. First let us 'double' $\hat{Q}$ in the following way: put $\overline{\hat{Q}}=\hat{Q} \cup \hat{Q}^{-1}$, where $\hat{Q}^{-1}$ is a disjoint copy of $\hat{Q}$ together with a fixed bijection ${ }^{-1}: \hat{Q} \rightarrow \hat{Q}^{-1}, q_{y z} \mapsto q_{y z}^{-1}$. Let $A^{-1}=\left\{a^{-1}: a \in A\right\}$, where

$$
a^{-1}= \begin{cases}\left(g x, x^{-1}\right) & \text { if } a=(g, x) \in G_{\mathcal{V}} \times X ; \\ \left(g p_{y z}, q_{y z}^{-1}\right) & \text { if } a=\left(g, q_{y z}\right) \in G_{\mathcal{V}} \times \hat{Q} .\end{cases}
$$

Notice that, for each edge $a \in A$, the edge $a^{-1}$ can be interpreted as the reverse of $a$, since the initial vertex of $a^{-1}$ is just the terminal vertex of $a$, the terminal vertex of $a^{-1}$ is just the initial vertex of $a$, and the label of $a^{-1}$ is the inverse of the label of $a$. Finally, observe that $\mathcal{X}$ can be obtained from the Cayley graph of $G_{\mathcal{V}}$ by adding loop edges to it at each vertex with labels $q_{y z}$ where $y$ or $z$ is $x_{0}$.

As it was mentioned above, the elements of $T$ are $\rho_{\mathcal{U}}$-classes of words over the alphabet $\bar{A}$. Observe that the words $r_{x}$ and $r_{x^{\prime}}$ chosen to represent the first components of $\tilde{x}$ and $\tilde{x}^{\prime}$, respectively, label paths on the graph $\mathcal{X}$ from 1 to the respective $G_{\mathcal{V}}$ components $m(x)$ and $m\left(x^{\prime}\right)$ (see Section 2).

The regular subsemigroup $W$ in $T \diamond, C$ generated by $\left\{\tilde{x}, \tilde{x}^{\prime}: x \in X\right\}$ can be obtained recursively with respect to the complexity of the terms in $\mathbf{T}(X)$. This leads to the following definition. As usual, for any $w \in \mathbf{T}(X)$, we denote the element of $C$ corresponding to $w$ by $(h(w), m(w), t(w))$.

Now we define an element $\omega(w)$ of $\mathbf{I}(A)$ for any term $w \in \mathbf{T}(X)$ as follows: for $x \in X$, let $\omega(x)=r_{x}$ and $\omega\left(x^{\prime}\right)=r_{x^{\prime}}$. Moreover, if $\omega(u)$ and $\omega(v)$ are defined for some $u, v \in \mathbf{T}(X)$, then let

$$
\begin{aligned}
\omega(u v) & =\omega(u)\left(m(u), q_{t(u) h(v)}\right) \cdot{ }^{m(u) p_{t(u) h(v)}} \omega(v) \quad \text { and } \\
\omega(u \wedge v) & =\omega(u) \omega(u)^{-1}\left(p_{t(v) h(u)}^{-1}, q_{t(v) h(u)}\right)^{-1} \cdot p_{l(v) h(u)}^{-1} m^{(v)^{-1}}\left(\omega(v)^{-1} \omega(v)\right) .
\end{aligned}
$$

It is easy to see by induction that, for every $w \in \mathbf{T}(X)$, the word $\omega(w) \in \mathbf{I}(A)$ is a path on $\mathcal{X}$ from 1 to $m(w)$. For any term $w \in \mathbf{T}(X)$, let us define

$$
w \iota=\left(h(w), \omega(w) \rho_{\mathcal{U}}, m(w), t(w)\right) \in T \diamond C .
$$


In particular, we have $x \iota=\tilde{x}$ and $x^{\prime} \iota=\tilde{x}^{\prime}$ for any $x \in X$. An induction on the complexity of terms shows that $\iota$ is a $(\cdot, \wedge)$-homomorphism. For, it is clear by definition that $u \iota \cdot v \iota=(u v) \iota$, and Lemma 4.2 implies that $u \iota \wedge v \iota=(u \wedge v) \iota$. Denote the image of $\iota$ by $R_{X}(\mathcal{U}, \mathcal{V})$. Since $R_{X}(\mathcal{U}, \mathcal{V})$ is just the regular subsemigroup of $T \diamond_{r} C$ generated by $\left\{\tilde{x}, \tilde{x}^{\prime}: x \in X\right\}$, Theorem 4.3 implies that, in this way, we have a model of the bifree object in $\mathcal{U} \diamond_{r} \mathcal{V}$ on $X$.

THEOREM 4.4. Let $\mathcal{U}$ be a variety of inverse semigroups and $\mathcal{V}$ a variety of completely simple semigroups with $\mathcal{R B} \subseteq \mathcal{V}$. For any non-empty set $X$, the subsemigroup

$$
R_{X}(\mathcal{U}, \mathcal{V})=\left\{\left(h(w), \omega(w) \rho_{\mathcal{U}}, m(w), t(w)\right): w \in \mathbf{T}(X)\right\}
$$

of $F \mathcal{U}(A) \diamond, B F \mathcal{V}(X)$ together with the matched mapping $\bar{X} \rightarrow R_{X}(\mathcal{U}, \mathcal{V}), y \mapsto$ $\left(h(y), \omega(y) \rho_{\mathcal{U}}, m(y), t(y)\right)(y \in \bar{X})$ is a bifree object in $\mathcal{U} \diamond_{r} \mathcal{V}$ on the set $X$.

Combining Theorem 4.4 with Theorem 3.9 and Theorem 3.10, we deduce the following corollary.

COROLLARY 4.5. Let $\mathcal{U}$ be a variety of inverse semigroups and $\mathcal{V}$ a variety of completely simple semigroups. Let $X$ be a non-empty set.

(i) If $\mathcal{R B} \subseteq \mathcal{V}$ then the semigroup $R_{X}(\mathcal{U}, \mathcal{V})$ is a bifree object in $\mathcal{U} *_{r} \mathcal{V}$ on the set $X$.

(ii) If $\mathcal{U}$ is non-trivial and $\mathcal{V}$ is a variety of right groups such that $\mathcal{V} \nsubseteq \mathcal{G}$ then the semigroup $R_{X}(\mathcal{U}, \mathcal{L} \mathcal{Z} \vee \mathcal{V})$ is a bifree object in $\mathcal{U} *_{r} \mathcal{V}$ on the set $X$

The arguments of this section apply with slight modifications to Pastijn products. Thus, if $\mathcal{U}$ is a variety of inverse semigroups and $\mathcal{V}$ a variety of completely simple semigroups with $\mathcal{R B} \subseteq \mathcal{V}$ then we obtain a model of a bifree object in the Pastijn product $\mathcal{U} \odot \mathcal{V}$ of $\mathcal{U}$ by $\mathcal{V}$, which coincides with the restricted regular semidirect product $\mathcal{U} *_{r r} \mathcal{V}$ of $\mathcal{U}$ by $\mathcal{V}$, in the following way. For any $w \in \mathbf{T}(X)$, define $\chi(w)$ to be the word in $\bar{A}$ obtained from $\omega(w)$ by deleting all factors $(g, q)$ with labels $q \in \overline{\hat{Q}}$. Notice that $\chi(w)$ does not label a path any more. Instead of $\mathcal{X}$, we can work with the subgraph of the Cayley graph of $G_{\mathcal{V}}$ consisting of the edges with labels in $\bar{X}$. In particular, $\chi(x)=(1, x)(1, x)^{-1}(1, x)$-which can be clearly changed for $\chi(x)=(1, x)$-and $\chi\left(x^{\prime}\right)=\left(p_{x x^{\prime}}^{-1} x^{-1}, x\right)^{-1}$. Thus we obtain the following result.

THEOREM 4.6. Let $\mathcal{U}$ be a variety of inverse semigroups and $\mathcal{V}$ a variety of completely simple semigroups with $\mathcal{R B} \subseteq \mathcal{V}$. For any non-empty set $X$, the subsemigroup

$$
P_{X}(\mathcal{U}, \mathcal{V})=\left\{\left(h(w), \chi(w) \rho_{\mathcal{U}}, m(w), t(w)\right): w \in \mathbf{T}(X)\right\}
$$

is a regular subsemigroup of the Pastijn product $F \mathcal{U}(A) \odot B F \mathcal{V}(X)$, and $P_{X}(\mathcal{U}, \mathcal{V})$ together with the matched mapping $\bar{X} \rightarrow P_{X}(\mathcal{U}, \mathcal{V}), y \mapsto\left(h(y), \chi(y) \rho_{\mathcal{U}}, m(y), t(y)\right)$ $(y \in \bar{X})$ is the bifree object in $\mathcal{U} *_{r r} \mathcal{V}=\mathcal{U} \odot \mathcal{V}$ on the set $X$. 
This construction was introduced in [10] in the special case of $\mathcal{U}$ being the variety $\mathcal{S}$ of semilattices to give models of bifree objects in the e-varieties of locally inverse semigroups generated by Malcev products $\mathcal{S} \circ \mathcal{V}$.

Finally, let us note that Theorems 4.4 and 4.6 have the following consequence. Given a (regular) Rees matrix semigroup $S=(\mathcal{R}) \mathcal{M}(I, U * G, \Lambda ; P)$ over a semidirect product of an inverse semigroup by a group, we can associate to it a completely simple semigroup $S \pi_{\mathcal{C S}}$ as follows: $S \pi_{\mathcal{C S}}=\mathcal{M}\left(I, G, \Lambda ; P^{\prime}\right)$, where the $(\lambda, i)$ entry of $P^{\prime}$ is the component from $G$ of the $(\lambda, i)$ entry of $P$.

COROLLARY 4.7. Let $\mathcal{U}$ be a variety of inverse semigroups and $\mathcal{V}$ a variety of completely simple semigroups with $\mathcal{R B} \subseteq \mathcal{V}$.

(i) The e-variety $\mathcal{U} *_{r} \mathcal{V}=\mathcal{U} \diamond_{r} \mathcal{V}$ is generated by the class of all regular Rees matrix semigroups $S=\mathcal{R} \mathcal{M}(I, U * G, \Lambda ; P)$, where $U \in \mathcal{U}$ and $S \pi_{\mathcal{C S}} \in \mathcal{V}$.

(ii) The e-variety $\mathcal{U} *_{r r} \mathcal{V}=\mathcal{U} \odot \mathcal{V}$ is generated by the class of all Rees matrix semigroups $S=\mathcal{M}(I, U * G, \Lambda ; P)$, where $U \in \mathcal{U}, S \pi_{\mathcal{C S}} \in \mathcal{V}$ and the entries of $P$ are in the group of units of $U^{1} * G$.

\section{References}

[1] K. Auinger, 'The bifree locally inverse semigroup on a set', J. Algebra 166 (1994), 630-650.

[2] K. Auinger and L. Polák, 'A semidirect product for locally inverse semigroups', Acta Sci. Math. 63 (1997), 405-435.

[3] K. Auinger and M. B. Szendrei, 'Comparing the regular and the restricted regular semidirect products', Algebra Universalis 51 (2004), 9-28.

[4] B. Billhardt and M. B. Szendrei, 'Associativity of the regular semidirect product of existence varieties', J. Austral. Math. Soc. Ser. A 69 (2000), 85-115.

[5] - 'Weakly E-unitary locally inverse semigroups', J. Algebra 267 (2003), 559-576.

[6] T. E. Hall, 'Identities for existence varieties of regular semigroups', Bull. Austral. Math. Soc. 40 (1989), 59-77.

[7] J. M. Howie, Fundamentals of semigroup theory (Clarendon Press, Oxford, 1995).

[8] P. R. Jones, 'Rees matrix covers and semidirect products of regular semigroups', J. Algebra 218 (1999), 287-306.

[9] P. R. Jones and P. G. Trotter, 'Semidirect products of regular semigroups', Trans. Amer. Math. Soc. 349 (1997), 4265-4310.

[10] J. Kađourek, 'On some existence varieties of locally inverse semigroups', Internat. J. Algebra Comput. 6 (1996), 761-788.

[11] — 'On some existence varieties of locally orthodox semigroups', Internat. J. Algebra Comput. 7 (1997), 93-131.

[12] J. Kađourek and M. B. Szendrei, 'On existence varieties of $E$-solid semigroups', Semigroup Forum 59 (1999), 470-521.

[13] T. A. Khan and M. V. Lawson, 'Variants of regular semigroups', Semigroup Forum 62 (2001), 358-374. 
[14] M. V. Lawson, Inverse semigroups: the theory of partial symmetries (World Scientific, Singapore, 1998).

[15] D. B. McAlister, 'Rees matrix semigroups and regular Dubreil-Jacotin semigroups', J. Austral. Math. Soc. Ser. A 31 (1981), 325-336.

[16] _- 'Rees matrix covers for locally inverse semigroups', Trans. Amer. Math. Soc. 277 (1983), $727-738$.

[17] F. Pastijn, 'The structure of pseudo-inverse semigroups', Trans. Amer. Math. Soc. 273 (1982), 631-655.

[18] M. Petrich, Inverse semigroups (Wiley \& Sons, New York, 1984).

[19] Y. T. Yeh, 'The existence of e-free objects in e-varieties of regular semigroups', Internat. J. Algebra Comput. 2 (1992), 471-484.

Fakultät für Mathematik

Universität Wien

Nordbergstrasse 15

A-1090 Wien

Austria

e-mail: karl.auinger@univie.ac.at
Bolyai Institute University of Szeged Aradi vértanúk tere 1 H-6720 Szeged Hungary e-mail: m.szendrei@ math.u-szeged.hu 